\section{ORIGINAL RESEARCH}

I. Oran

C. Cinar

\title{
Continuous Intra-Arterial Infusion of Nimodipine During Embolization of Cerebral Aneurysms Associated With Vasospasm
}

\begin{abstract}
BACKGROUND AND PURPOSE: Despite rigorous efforts, cerebral vasospasm remains an important cause of morbidity and mortality in patients who survive their initial subarachnoid hemorrhage. In cases of intracranial ruptured aneurysm associated with vasospasm, we evaluated the effectiveness of combined embolization of an aneurysm and intra-arterial infusion of nimodipine, which continued during the entire procedure.
\end{abstract}

\begin{abstract}
MATERIALS AND METHODS: Ten patients with ruptured aneurysms associated with vasospasm who were treated in a single session were reviewed retrospectively. After initial intra-arterial infusion of nimodipine (1-2 mg within 10-15 minutes), they underwent occlusion of the aneurysm with coils under continuous intra-arterial infusion of nimodipine at a dose of $1 \mathrm{mg}$ per hour.
\end{abstract}

\begin{abstract}
RESULTS: Angiography showed severe proximal vasospasm in 1 patient, proximal and distal in 3 patients, and distal in 3 patients. There was also moderate proximal vasospasm in 4 patients, proximal and distal in 1 patient, and distal in 1 patient. Complete occlusion of the aneurysm was achieved in 5 patients, incomplete occlusion in 3 patients, and a small neck remnant in 2 patients. Final angiograms also demonstrated complete clearance of a proximal spasm in 4 patients, and complete clearance of proximal and distal spasms in another 4 patients. Mean initial dose of nimodipine was $1.375 \mathrm{mg}$, and mean continuous infusion dose was $1.275 \mathrm{mg}$ (mean total dose, $2.65 \mathrm{mg}$ ). No medical complications related to extended infusion of nimodipine occurred.
\end{abstract}

CONCLUSION: In this small series, extended intra-arterial infusion of nimodipine up to the end of the embolization procedure was effective and safe in patients with a ruptured aneurysm and associated vasospasm. This technique seems to increase the security of the procedure as well as force further vasorelaxation when the endovascular route is used to treat both the aneurysm and vasospasm in a single step.

$\mathbf{T}$ he current optimized approach for patients with a ruptured intracranial aneurysm is to secure the aneurysm early (usually within 3-4 days), either surgically or endovascularly, then apply triple- $\mathrm{H}$ therapy alone or in combination with intra-arterial chemical or mechanical angioplasty to overcome vasospasm if it is apparent clinically. ${ }^{1}$ In cases of an aneurysm of a high clinical grade or accompanying medical problems that preclude an open surgical procedure, in patients who are candidates for open surgery initially but cannot be operated on later for any reason, and because of other obstacles (ie, geographic distance to referral center) that delay initial intervention, an endovascular interventionalist may encounter an aneurysm along with vasospastic cerebral arteries.

Here we describe a simple technique that involves both procedures of aneurysmal embolization and intra-arterial infusion of a vasorelaxing drug in such patients with significant vasospasms.

\section{Methods}

In cases of patients who have vasospasms related to a subarachnoid hemorrhage (SAH), we have used nimodipine instead of papaverine intra-arterially for 3 years. During the same period, all patients with ruptured intracranial aneurysms associated with significant arterial vasospasm were treated with coil embolization with the aid of a va-
\end{abstract}

Received May 31, 2007; accepted after revision August 8.

From the Ege University Medical School Department of Radiology, Izmir, Turkey.

Please address correspondence to Ismail Oran, MD, Ege University Hospital, Department of Radiology, 35100 Izmir, Turkey. E-mail: ismailoran@gmail.com

DOI 10.3174/ajnr.A0830 sorelaxing effect of continuous intra-arterial infusion of nimodipine. We retrospectively reviewed 10 such patients. The age of the patients was 35 to 79 years old (average age, 49 years); 7 were women and 3 were men. Hunt and Hess grade was III in 6 patients, IV in 2, and V in 2 patients at the time of treatment. On CT, the initial SAH grade was Fisher III or IV for all but 2 patients. The accompanying table shows the location of the aneurysms, spastic vessels, and clinical course.

As in previous studies, ${ }^{2,3} 2$ neuroradiologists semiquantitatively and subjectively assessed a pretreatment spasm. The spasm was graded as mild when arterial narrowing was less than $25 \%$, moderate when $25 \%$ to $50 \%$ constriction was present, and severe when the narrowing was more than $50 \%$. The initial admission angiogram that was used for comparison to assess the vasospasms was available in only 5 patients (patients $4,5,8,9$, and 10). In 2 other patients (patients 1 and 3), follow-up angiograms after embolization (10 and 12 months, respectively) were used as a reference and were compared with pretreatment angiograms to assess the degree of vasospasm. Patient 7 had a focal spasm just before an aneurysm of the anterior communicating artery (AcomA), at the distal end of the A1 segment of the anterior cerebral artery. Because there was no obvious evidence of a spasm in other areas of the cerebral arterial circulation, we assessed the degree of vasospasm using a diameter measurement of the distal focal spastic area and proximal nonspastic segment of the A1 artery. In the last 2 patients (patients 2 and 6), the vasospasm could be assessed with the use of pretreatment and posttreatment angiograms only; therefore, it was vulnerable to subjectivity. Both neuroradiologists separately evaluated all angiograms and then compared their findings. The degree of vasospasm was assessed by calculating the mean of 2 separate measurements. In cases of disagreement of the 


\begin{tabular}{|c|c|c|c|c|c|c|c|c|c|c|c|c|}
\hline \multirow{2}{*}{$\begin{array}{l}\text { No./Age/ } \\
\text { Sex }\end{array}$} & \multirow[b]{2}{*}{ Aneurysm } & \multirow{2}{*}{$\begin{array}{c}\mathrm{HH} \\
\text { Grade }\end{array}$} & \multirow{2}{*}{$\begin{array}{l}\text { Days } \\
\text { After } \\
\text { SAH }\end{array}$} & \multirow{2}{*}{$\begin{array}{c}\text { Vasospasm } \\
\text { Symptom }\end{array}$} & \multirow[b]{2}{*}{ Spastic Segment } & \multicolumn{2}{|c|}{ Vasospasm } & \multicolumn{2}{|c|}{$\begin{array}{c}\text { Arterial } \\
\text { Nimodipine } \\
\text { (mg) }\end{array}$} & \multirow{2}{*}{$\begin{array}{c}\text { Aneurysmal } \\
\text { Occlusion }\end{array}$} & \multirow[b]{2}{*}{$\mathrm{mRS}$} & \multirow{2}{*}{$\begin{array}{l}\text { Follow-up } \\
\text { (yrs/mos) }\end{array}$} \\
\hline & & & & & & Before & After & Bolus & Infusion & & & \\
\hline $1 / 55 / F$ & Basilar tip & 3 & 8 & + & BA, Bilateral diffuse ICA & Mo, Se & $\mathrm{Co},{ }^{*}$ & 1 & 0.75 & Incomplete & 1 & 3 yrs \\
\hline $2 / 45 / F$ & PcomA & 4 & 7 & + & RICA, RA1-A2, RM1-M2 & Mo, Se, Se & Co, Mi, Mi & 2 & 1.5 & Neck remnant & 3 & $2 y r s$ \\
\hline $3 / 50 / F$ & Ophthalmic & 3 & 7 & + & RICA, RM1 & $\mathrm{Se}, \mathrm{Se}$ & Mo, Mi & 2 & 1 & Complete & 3 & 3 yrs \\
\hline $4 / 35 / F$ & Basilar tip & 3 & 6 & - & BA, bilateral P1-P2 & Mo, Se & $\mathrm{Co}, \mathrm{Mi}$ & 1 & 1.75 & Neck remnant & 2 & $6 \mathrm{mos}$ \\
\hline 5/65/M & AcomA & 5 & 14 & + & Bilateral $\mathrm{A} 1$ and $\mathrm{A} 2$ & Mo & Co & 1 & 1 & Incomplete & Died & - \\
\hline 6/79/F & AcomA & 3 & 9 & + & LA1, bilateral A2 & $\mathrm{Se}, \mathrm{Se}$ & Co, Co & 1.75 & 1 & Incomplete & 2 & $1 \mathrm{yr}$ \\
\hline 7/45/M & AcomA & 4 & 4 & - & LA1, LA2 & Se, Mi & Co, Co & 1.5 & 1 & Complete & 3 & $6 \mathrm{mos}$ \\
\hline 8/35/M & AcomA & 5 & 11 & + & LA1, bilateral A2 & Mo, Mo & Co, Mi & 1 & 1.25 & Complete & Died & - \\
\hline 9/42/F & SCA & 3 & 8 & - & BA, bilateral P1-P2 & $\mathrm{Se}, \mathrm{Se}$ & Mo, Mo & 1 & 1.5 & Complete & 2 & $6 \mathrm{mos}$ \\
\hline 10/38/F & AchoA & 3 & 13 & + & LICA, LA1-A2, LM1-M2 & Mo, Se, Se & Co, Mi, Mi & 1.5 & 2 & Complete & 3 & $6 \mathrm{mos}$ \\
\hline
\end{tabular}

Note:- - H indicates Hunt and Hess; SAH, subarachnoid hemorrhage; mRS, Modified Rankin Score; BA, basilar artery; AcomA, anterior communicating artery; PcomA, posterior communicating artery; SCA, superior cerebellar artery; AchoA, anterior choroidal artery; ICA, internal carotid artery; LICA, left internal carotid artery; RICA, right internal carotid artery; Mi, mild; Mo, moderate; Se, severe; Co, complete dilation $(<10 \%$ spasm)

* Not determined.

grade of the spasm, another neuroradiologist blinded to the findings of both readers evaluated the angiograms, and disagreement in grading was resolved by consensus. To minimize observer bias, all measurements were obtained with the use of an electronic cursor on a digital image. In each case, the point of maximum vasospasm was used as the reference point for calculation. During comparison of angiograms, we accounted for magnification differences by comparing ratios of the diameter of the vessel involved with that of the nearest extradural main arterial trunk (internal carotid or vertebral artery).

We performed all procedures with the patients under general anesthesia. Intravenous bolus of heparin 5000U was administered at the start, followed by $1000 \mathrm{U}$ every hour until completion. After insertion of $6 \mathrm{~F}$ guiding catheter near to or in the petrous segment of the internal carotid artery, or in the upper cervical portion of the vertebral artery, we obtained an initial angiogram to judge the endovascular strategy. The ruptured aneurysm, along with the vasospasm, was a common finding on angiograms. At the beginning, the neuroanesthesiologist increased the rate of intravenous fluid replacement to maintain a main arterial blood pressure of more than $85 \mathrm{~mm} \mathrm{Hg}$. Subsequently, 1 to $2 \mathrm{mg}$ of nimodipine (diluted in a $25-\mathrm{mL}$ syringe) was infused intra-arterially by manual injection through the guiding catheter within 10 to 15 minutes. During this initial infusion of nimodipine, the systolic arterial pressure was not allowed to drop more than 15 $\mathrm{mm} \mathrm{Hg}$. When this happened, we slowed down or stopped the infusion for a short period of time. We did not perform direct monitoring of intracranial pressure. Therefore, we searched for indirect signs of increased intracranial pressure (ie, rise in blood pressure, bradycardia) using continuous monitoring of blood pressure and heart rate. Before the insertion of a microcatheter, we connected an electric pressure pump to a guiding catheter and were prepared to give nimodipine $1 \mathrm{mg}$ per hour intra-arterially. With road-mapping, we placed an Excelsior microcatheter (Boston Scientific, Natick, Mass) into the aneurysm and accomplished deposition of the coil (GDC; Boston Scientific, Cork, Ireland or Microplex; MicroVention, Aliso Viejo, Calif) in the usual way. When necessary, a $4-\times 7$-mm HyperForm intracranial balloon (ev3, Irvine, Calif) was used as a complementary tool.

\section{Results}

Besides an aneurysm responsible for SAH, a diffuse spasm proximal to the aneurysm and neck region and/or distal to the aneurysm was observed in all but 1 patient (Table). In patient 3 , a vasospasm was located in the arteries distal to the aneu- rysm only. A vasospasm was so intense that it was judged risky to pass through with the microcatheter or remain lodged in the proximal spastic parent artery during the entire procedure in 6 of 10 patients. The mean initial bolus dose of nimodipine was $1.375 \mathrm{mg}$ (range, 1 to $2 \mathrm{mg}$ ). In 2 patients (patients 4 and 9), the systolic blood pressure was apt to drop more than 15 $\mathrm{mm} \mathrm{Hg}$ during the initial infusion (it never exceeded $15 \mathrm{~mm}$ $\mathrm{Hg}$ ). Therefore we stopped the manual nimodipine infusion for a short period of time (not longer than 3 minutes) as the neuroanesthesiologist further increased the rate of intravenous fluid infusion. With these maneuvers (ie, cessation of nimodipine infusion and increasing intravenous fluid replacement), the initial dose of nimodipine was infused within 10 to 15 minutes in these 2 patients as in the remaining 8 patients (Table).

Control angiograms obtained immediately after completion of initial infusion of nimodipine showed some degree of relaxation in all patients. However, we observed at least 1 degree of improvement (ie, severe to mild or mild to moderate) in patients 10 and 2 (Figs 1,2). Subsequently, microcatheterization was accomplished without technical difficulty, and embolization was done as usual. According to the results of the control angiograms, the degree of aneurysmal occlusion was considered to be sufficient, so we stopped the procedure in all but 1 patient. In patient 4 , sufficient occlusion could not be achieved because of a large aneurysmal neck. An intracranial balloon was inserted through the same guiding catheter and positioned across the neck. We then sufficiently occluded the large-neck basilar tip aneurysm using a balloon remodeling technique, which extended the total time of the procedure to 1.75 hours. When the procedure was finished 1 to 2 hours after starting the second infusion of nimodipine (via an electric pump), control angiograms showed complete clearance of the proximal spasm in 4 patients, and complete proximal and distal clearance in the other 4 patients (Figs 1 and 2). Eventually, we obtained 5 aneurysms that were occluded completely, 3 aneurysms that were occluded incompletely, and 2 aneurysms with a neck remnant.

During recovery from the anesthesia, patient 1 was also treated with another dose of nimodipine $1 \mathrm{mg}$ per 10 minutes intra-arterially through each carotis because of additional bilateral diffuse vasospasms in the internal carotid artery (Ta- 

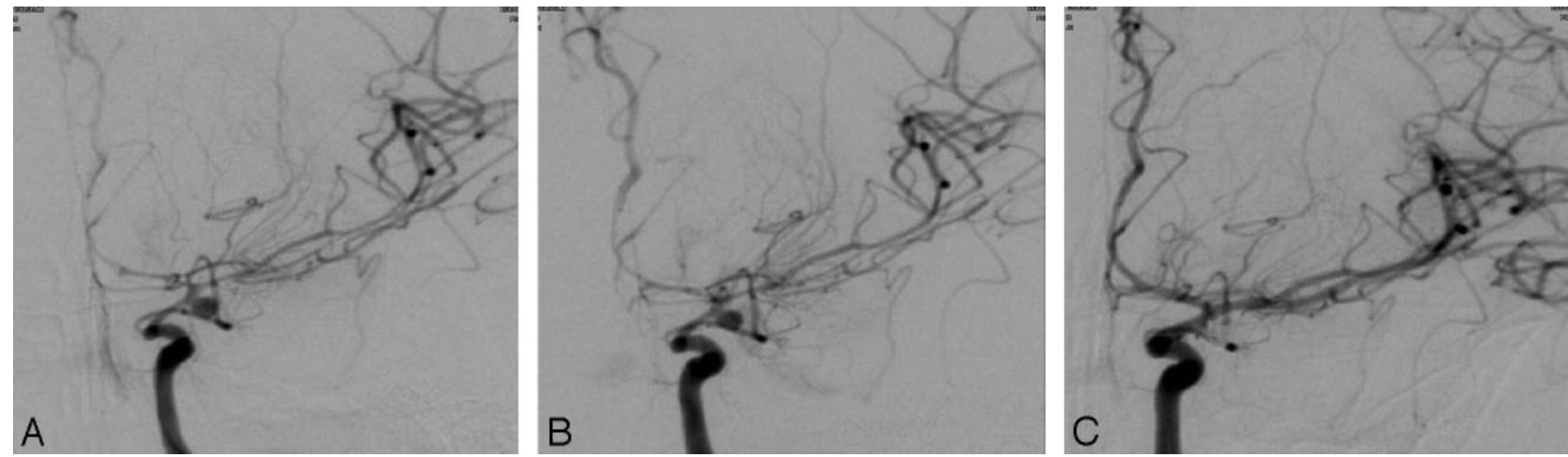

Fig 1. Patient 10: an aneurysm of the left anterior choroidal artery. Angiogram obtained at anterior-posterior projection $(A)$ shows a diffuse vasospasm involving the terminal internal carotid artery (ICA), the $\mathrm{A} 1$ and $\mathrm{A} 2$ segments of the anterior cerebral artery (ACA), and the M1 and M2 segments of the middle cerebral artery (MCA). Ten minutes later, after intra-arterial infusion of $1.5 \mathrm{mg}$ of nimodipine, an angiogram at the same projection $(B)$ shows slight improvement but still diffuse vasospasm. After the end of the embolization, under continuous nimodipine infusion at the dose of $1 \mathrm{mg}$ per hour (total, $2 \mathrm{mg}$ ), an angiogram $(C)$ shows significant vasorelaxation.
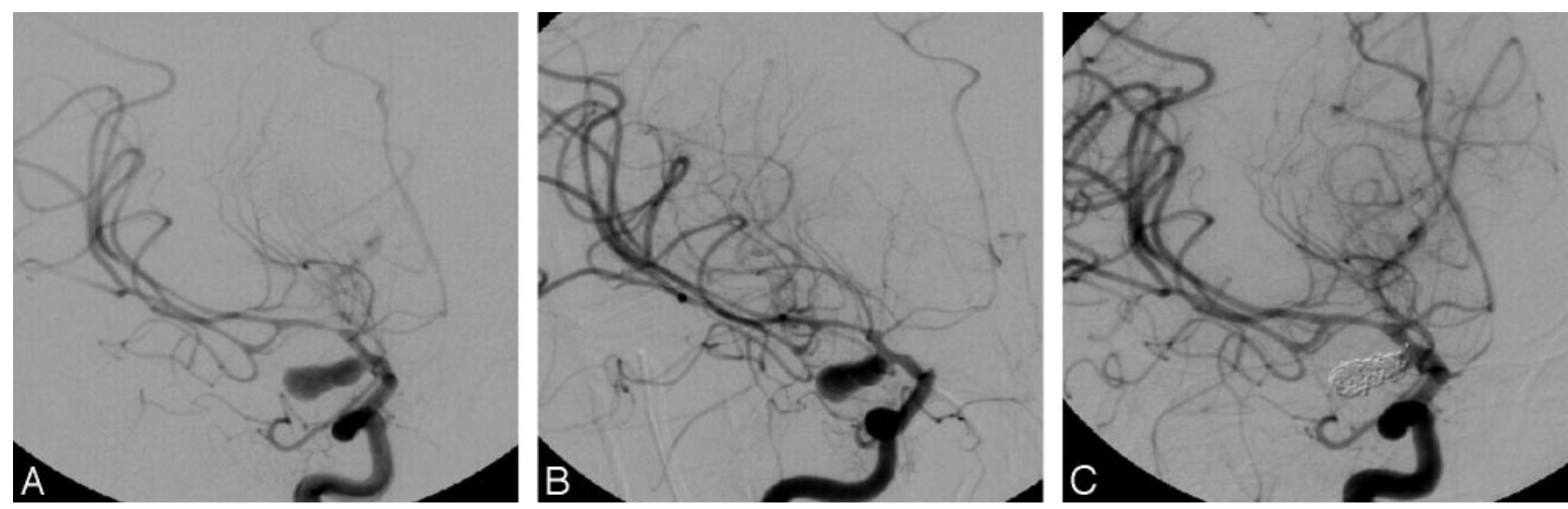

Fig 2. Patient 2: an aneurysm of the right posterior communicating artery. An angiogram obtained at the right anterior oblique projection ( $A$ ) shows a vasospasm at the supraclinoid ICA the $\mathrm{A} 1$ and $\mathrm{A} 2$ segments of the ACA, and the M1 and M2 segments of the MCA. Fourteen minutes later, after intra-arterial infusion of 2 mg of nimodipine, this angiogram (B) shows some degree of vasorelaxation, especially in the proximal segments of the ACA. Final angiogram at the same projection after completion of embolization (C), with continuous intra-arterial infusion of $1.5 \mathrm{mg}$ of nimodipine throughout the procedure, demonstrates complete disappearance of the proximal vasospasm and near-complete clearance of the distal spasm as well as occlusion of the aneurysm.

ble). There were no technical complications during the aneurysmal embolization. No apparent side effects were observed either on blood pressure or heart rate during intra-arterial infusion of nimodipine, which lasted approximately 1 to 2 hours at a dose of $1 \mathrm{mg}$ per hour (mean, $1.275 \mathrm{mg}$ ). The mean total dose of nimodipine at the end of the embolization was $2.65 \mathrm{mg}$ per vessel (range, 1.75-3.5 mg). Of 10 patients, 2 died within 1 month primarily because of the early brain injury related to $\mathrm{SAH}$ and accompanying medical problems. The remaining 8 patients remained stable or improved gradually and were discharged eventually. No patients developed new clinical evidence of vasospasm during the remainder of their hospital course. Control angiograms at 1 year were available in 2 of 8 patients. Patient 3, who had a complete aneurysmal occlusion initially, showed persistence of the occlusion on control angiograms. Patient 1 , who had an initial incomplete aneurysmal occlusion, showed complete occlusion on control angiograms. Clinical follow-up ranged from 6 months to 3 years for the 8 patients, and rebleeding did not occur.

\section{Discussion}

Although early intervention (within 3-4 days) for ruptured intracranial aneurysms has gained favor in recent years, it is not always possible in every patient for various reasons. In late cases, if the treatment is via the endovascular route, a vasospasm will be encountered frequently. A vasospasm is also a big problem even in the early period of a $\mathrm{SAH}$, especially in patients with high-grade aneurysms. During early endovascular treatment of an aneurysm in patients with Hunt and Hess grade IV and V, angiographic evidence of a vasospasm was found in $92 \%$ and $51.4 \%$ of all patients, and $26 \%$ and $36.9 \%$ of these required endovascular treatment of the vasospasm, respectively. ${ }^{4,5}$ In cases of an unsecured aneurysm and symptomatic vasospasm, endovascular treatment seems to be a reasonable therapeutic option with achievement of single-session aneurysmal occlusion and vasorelaxation. ${ }^{6,7}$ These frequently used techniques aim to achieve arterial dilation by means of mechanical balloon angioplasty and local intra-arterial administration of vasorelaxing drugs. Nimodipine, a calcium antagonist, has recently been demonstrated to be safe and effective when administered intra-arterially for the treatment of a cerebral vasospasm. ${ }^{3,8,9}$ Indeed, prophylactic oral or intravenous use of this drug after SAH has long been known. A typical intra-arterial infusion dose of nimodipine per vessel is 1 to 2 mg during 10 to 15 minutes (total dose not exceeding $4 \mathrm{mg}$ ). 3,9 Notable vascular dilation was observed in $43 \%$ and nearly $100 \%$ of vessels were treated, respectively. ${ }^{3,9}$

Microcatheterization through the spastic vessel may be dif- 
ficult or even impossible. ${ }^{6,7}$ When the parent artery of the aneurysm is already critically narrowed, it is necessary to open the parent artery first to gain access into the aneurysm. ${ }^{7} \mathrm{Be}-$ sides an effort to relieve the spasm, the parent artery may not be fully opened, and the microcatheter remaining in a wedge position for a considerable amount of time may eventually induce thromboembolic complications during aneurysmal embolization. Murayama et $\mathrm{al}^{7}$ have encountered 1 such complication resulting from microcatheter obstruction in a series of 12 patients who underwent combined endovascular treatment of both an aneurysm and a vasospasm. We started with the same infusion dose of nimodipine just after placement of a guiding catheter in all of our patients; microcatheterization was done afterward. Intra-arterial infusion of nimodipine through the guiding catheter continued throughout the entire procedure at a rate of $1 \mathrm{mg}$ per hour.

One possible objection may have been the dose of intraarterial nimodipine in our patients with already lowered blood pressure resulting from general anesthesia. Indeed, during the initial manual infusion, the systolic blood pressure was apt to drop more than $15 \mathrm{~mm} \mathrm{Hg}$ in 2 patients; so the infusion was stopped for nearly 3 minutes. On the other hand, the continuous nimodipine infusion at a dose of $1 \mathrm{mg}$ per hour did not cause any changes in blood pressure. Actually, the manufacturer recommends nimodipine at $2 \mathrm{mg}$ per hour for the intravenous route. We preferred half of the recommended dose for intra-arterial infusion and used this dose for up to 2 hours without apparent cardiovascular complication. With this technical modification of nimodipine infusion, our aim was to obtain further relaxation of the spastic vessels, thus reducing the possible risk of complication. Indeed, the control angiograms obtained at the end of embolization showed complete clearance of a proximal spasm in 4 patients, and proximal and distal spasms in the other 4 patients. There was significant vasorelaxation in all other spastic vessels as well (Table).

However, the results of the angiograms obtained immediately after initial infusion of nimodipine were not satisfactory in our study population (Figs 1 and 2) compared with those of the previous 2 studies. ${ }^{3,9}$ We concluded that these results were mainly because of the dose of nimodipine and the time to control angiograms. We determined the initial dose of nimodipine by more strict criteria when compared with previous studies; as a result, our initial dose of $1.375 \mathrm{mg}$ per vessel ( 1 to $2 \mathrm{mg}$ ) was well below that of previous studies. The intra-arterial infusion dose was $1.9 \mathrm{mg}$ per vessel (up to $3 \mathrm{mg}$ ) in $1 \mathrm{study}^{3}$ and $3.3 \mathrm{mg}$ per vessel (up to $4 \mathrm{mg}$ ) in another study. ${ }^{9}$ In previous studies, the control angiogram to assess the result was repeated 10 minutes $^{3}$ and 15 minutes ${ }^{9}$ after the end of the intra-arterial injection. On the other hand, we did not wait for control angiograms; instead, we obtained them just after the end of the intra-arterial infusion and then went on to perform the embolization. Therefore, we obtained less striking vasorelaxation on spastic vessels.

As a vasorelaxing substance, nimodipine intra-arterially administered into the intracranial circulation may increase intracranial pressure by fluid extravasation in the brain or decrease blood pressure by a relaxant effect on the systemic circulation. Both consequences have the potential risk of lowering cerebral perfusion pressure with subsequent ischemia in especially vasospastic areas. Direct intracranial pres- sure monitoring with a ventricular or lumbar catheter is an ideal strategy to exclude this risk. As in our institution, however, direct intracranial pressure monitoring is not a routine practice in all SAH patients. In a similar fashion, 2 clinical studies using intra-arterial infusion of nimodipine for vasospasms have not monitored direct intracranial pressure routinely, ${ }^{3,9}$ except for patients who already have had a ventricular drainage catheter. ${ }^{3}$ Routine indirect neurophysiologic monitoring, including continuous measurement of arterial blood pressure, central venous pressure, and heart rate, is therefore mandatory in such conditions. ${ }^{3,9}$ The most important one is blood pressure monitoring because the main significant systemic side effect of nimodipine is hypotension. Biondi et $\mathrm{al}^{3}$ have determined a critical drop in systolic blood pressure at 40 $\mathrm{mm} \mathrm{Hg}$; they have suspended infusion of nimodipine when the blood pressure dropped more than $40 \mathrm{~mm} \mathrm{Hg}$. In our study, we have determined this critical value more strictly (systolic pressure of $15 \mathrm{~mm} \mathrm{Hg}$ ) because our patients have already lowered blood pressure resulting from general anesthesia.

One other speculation in our study may have been the timing (namely before or during aneurysmal occlusion) of intraarterial vasorelaxing infusion. Aggressive triple- $\mathrm{H}$ therapy is generally not recommended to overcome a vasospasm in patients with an untreated ruptured aneurysm because of the risk for a recurrent rupture. ${ }^{1}$ Therefore, the interventionalist may hesitate to apply intra-arterial vasorelaxing infusion before securing the aneurysm. ${ }^{6}$ Although there is a tendency not to start treatment of an intra-arterial vasospasm before aneurysmal occlusion, ${ }^{6,7}$ sometimes it is inevitable. ${ }^{7}$ There is no evidence in the literature showing an increased risk for a recurrent aneurysmal rupture when an intra-arterial vasorelaxing drug (not balloon angioplasty) is used before aneurysmal occlusion. In the authors' opinion, pretreatment with intra-arterial nimodipine may be feasible for several reasons. First, aneurysmal embolization is usually performed with the patient under general anesthesia. General anesthesia lowers blood pressure and thus reduces the risk for a recurrent aneurysmal rupture during infusion of nimodipine. Second, intra-arterial infusion of nimodipine exerts its vasorelaxing effect in a progressive manner (not abrupt vasodilation as in the balloon angioplasty). It generally achieves partial vasorelaxation at the end of the initial infusion. Third, additional infusion of nimodipine throughout the embolization procedure may result in further (maybe full) vasorelaxation (as in our study), but coils already accumulated within the aneurysm would have increased the security of the aneurysm. Fourth, when the parent artery of the aneurysm is already spastic, microcatheterization through these segments and aneurysmal embolization in occlusive conditions may suggest a thromboembolic complication. This may necessitate thrombolysis, which carries a substantial risk for catastrophic rebleeding even in aneurysms that are filled with coils. To avoid these consequences, it is feasible to open the parent artery with intra-arterial infusion of nimodipine first and then begin embolization while continuing with the infusion.

In conclusion, intra-arterial infusion of nimodipine seems to be safe and effective in the treatment of a cerebral vasospasm. Throughout the entire procedure, it seems to be a simple, safe, and effective technical modification to obtain further vasorelaxation in the endovascular treatment of such chal- 
lenging cases of patients who have a combination of an aneurysm and a vasospasm. Further large, comparative series on procedural complication rates and clinical consequences are needed to find the real benefits.

\section{References}

1. Jahromi BS, Macdonald RL. Vasospasm: diagnosis and medical management. In: Le Roux PD, Winn HR, Newell DW, eds. Management of cerebral aneurysm. Philadelphia: Saunders; 2004: 455-87

2. Schuknecht B, Fandino J, Yüksel C, et al. Endovascular treatment of cerebral vasospasm: assessment of treatment effect by cerebral angiography and transcranial colour Doppler sonography. Neuroradiology 1999;41:453-62

3. Biondi A, Ricciardi GK, Puybasset L, et al. Intra-arterial nimodipine for the treatment of symptomatic cerebral vasospasm after aneurysmal subarachnoid hemorrhage: preliminary results. AJNR Am J Neuroradiol 2004;25: 1067-76
4. Weir RU, Marcellus ML, Do HM, et al. Aneurysmal subarachnoid hemorrhage in patients with Hunt and Hess grade 4 or 5: treatment using the Guglielmi detachable coil system. AJNR Am J Neuroradiol 2003;24:585-90

5. Suzuki S, Jahan R, Duckwiler GR, et al. Contribution of endovascular therapy to the management of poor-grade aneurysmal subarachnoid hemorrhage: Clinical and angiographic outcomes [published erratum appears in J Neurosurg 2007;106:204]. J Neurosurg 2006;105:664-70

6. Wanke I, Dörfler A, Dietrich U, et al. Combined endovascular therapy of ruptured aneurysms and cerebral vasospasm. Neuroradiology 2000;42:926-29

7. Murayama Y, Song JK, Uda K, et al. Combined endovascular treatment for both intracranial aneurysm and symptomatic vasospasm. AJNR Am J Neuroradiol 2003;24:133-39

8. Firat MM, Gelebek V, Orer HS, et al. Selective intraarterial nimodipine treatment in an experimental subarachnoid hemorrhage model. AJNR Am J Neuroradiol 2005;26:1357-62

9. Hui C, Lau KP. Efficacy of intra-arterial nimodipine in the treatment of cerebral vasospasm complicating subarachnoid hemorrhage. Clin Radiol 2005;60: $1030-36$ 